DRAFT VERSION SEPTEMBER 26, 2018

Preprint typeset using LTEX style emulateapj v. 08/22/09

\title{
KLEIN-NISHINA EFFECTS ON THE HIGH-ENERGY AFTERGLOW EMISSION OF GAMMA-RAY BURSTS
}

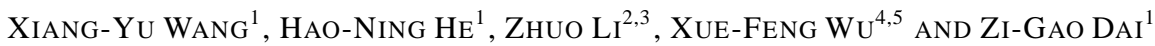 \\ Draft version September 26, 2018
}

\begin{abstract}
Extended high-energy $(\gtrsim 100 \mathrm{MeV})$ gamma-ray emission that lasts much longer than the prompt sub-MeV emission has been detected from quite a few gamma-ray bursts (GRBs) by Fermi Large Area Telescope (LAT) recently. A plausible scenario is that this emission is the afterglow synchrotron emission produced by electrons accelerated in the forward shocks. In this scenario, the electrons that produce synchrotron high-energy emission also undergo inverse-Compton (IC) loss and the IC scattering with the synchrotron photons should be in the Klein-Nishina regime. Here we study effects of the Klein-Nishina scattering on the high-energy synchrotron afterglow emission. We find that, at early times the Klein-Nishina suppression effect on those electrons that produce the high-energy emission is usually strong and therefore their inverse-Compton loss is small with a Compton parameter $Y \lesssim$ a few for a wide range of parameter space. This leads to a relatively bright synchrotron afterglow at high energies that can be detected by Fermi LAT. As the Klein-Nishina suppression effect weakens with time, the inverse-Compton loss increases and could dominate over the synchrotron loss in some parameter space. This will lead to a faster temporal decay of the high-energy synchrotron emission than what is predicted by the standard synchrotron model, which may explain the observed rapid decay of the early high-energy gamma-ray emission in GRB090510 and GRB090902B.
\end{abstract}

Subject headings: gamma rays: bursts

\section{INTRODUCTION}

With the launch of Fermi satellite, one of the new features of gamma-ray bursts at high-energies has been established, i.e. GRBs show extended high-energy ( $\gtrsim 100 \mathrm{MeV})$ emission which lasts much longer than the prompt phase. This extended emission has been seen in both long and short GRBs and the flux usually decays with time after the initial peak. In some cases (e.g. GRB090510 and GRB090902B), the temporal decay is a simple power-law decay with a slope ranging from -1.3 to -1.5 (Abdo et al. 2009a,b; Ghirlanda, Ghisellini, Nava 2009; De Pasquale et al. 2009). One of the proposed models for such emission is the hadronic cascade emission model, in which the high-energy photons produced by the accelerated ultra-high energy protons can not escape the soft photon field and a cascade is induced (Abdo et al. 2009b). This model has been applied to the extended emission of GRB941017 (Dermer \& Atoyan 2004), but whether it can explain the simple power-law decay of the Fermi LAT bursts is unknown. The long-lived behavior and not very rapid decay of the high-energy emission from GRB090510 and GRB090902B can not be easily explained by the reverse shock emission model either (Wang et al. 2001a,b) ${ }^{6}$. On the other hand, the simple power-law decay with a modestly large slope is reminiscent of the afterglow emission. The self inverse-Compton (IC) emission of the afterglow has been long

\footnotetext{
${ }^{1}$ Department of Astronomy, Nanjing University, Nanjing 210093, China

${ }^{2}$ Department of Astronomy, Peking University, Beijing 100871, China

${ }^{3}$ Kavli Institute for Astronomy and Astrophysics, Peking University, Beijing 100871, China

${ }^{4}$ Department of Astronomy and Astrophysics, Pennsylvania State University, 525 Davey Lab, University Park, PA 16802

${ }_{5}^{5}$ Purple Mountain Observatory, Chinese Academy of Sciences, Nanjing 210008, China

${ }^{6}$ The decay of the high-energy emission in GRB080825C is steeper than $t^{-1.7}$ (Abdo et al. 2009c), which is consistent with the synchrotron selfinverse Compton scattering emission from the reverse shock or cross IC scatterings between the reverse shock and forward shock (Wang et al. 2001a,b; Granot \& Guetta 2003; Pe'er \& Waxman 2004; Wang et al. 2005).
}

thought to produce a high-energy component (e.g. Zhang \& Mészáros 2001; Sari \& Esin 2001; Fan et al. 2008; Gou \& Mészáros 2007 ), but the light curve is expected to rise initially and start to decay minutes to hours after the burst. Kumar \& Barniol Duran (2009a) proposed that the extended high-energy emission from GRB080916C is due to afterglow synchrotron emission. This mechanism has also been proposed to explain the extended high-energy emission from GRB090510 (Gao et al. 2009; Ghirlanda, Ghisellini, Nava 2009; Ghisellini, Ghirlanda, Nava 2009, De Pasquale et al. 2009) and GRB090902B (Kumar \& Barniol Duran 2009b).

In the latter synchrotron afterglow scenario, the highenergy extended emission is produced by the electrons in the forward shock via synchrotron emission. The shockaccelerated electrons are usually assumed to have a power-law form in energy distribution, i.e. $d N_{e} / d \gamma_{e} \propto \gamma_{e}^{-p}$, where $\gamma_{e}$ is the Lorentz factor of electrons. These electrons also suffer IC loss by scattering synchrotron photons. Due to that the scattering between large $\gamma_{e}$ electrons and the synchrotron photons could enter the Klein-Nishina $(\mathrm{KN})$ scattering regime, higher energy electrons may suffer smaller IC loss and as a consequence, their synchrotron emission is stronger. Since the Lorentz factor of the electrons producing the high-energy afterglow emission are usually large, the KN scattering effect must be taken into account when one calculate the synchrotron high-energy afterglow emission. In this paper, we study the effect of the KN scattering on the high-energy afterglow emission of GRBs. Recently, Wang et al.(2009) studied the KN effect on the prompt emission spectrum of GRBs and Nakar et al. (2009) studied the KN effect on optically thin synchrotron and synchrotron self-Compton spectrum in general. In this paper, we focus on the early high-energy afterglow emission and confront the theoretical results with the high-energy afterglow observations by Fermi LAT.

The paper is organized as follows. First we study how the KN scattering affects the electron distribution in the forward shock in $\S 2$. Then in $\S 3$ we calculate the Compton 
parameters for the high-energy electrons that produce highenergy gamma-ray emission and study their evolution with time. With the Compton parameters known, we calculate the light curves of high-energy afterglows in $\S 3$. Finally, we summarize our findings in In $\S 4$.

\section{KLEIN-NISHINA EFFECT ON THE ELECTRON DISTRIBUTION}

We define the Compton parameter for electrons with Lorentz factor $\gamma_{e}$ as the ratio of the synchrotron self-inverse Compton (SSC) to the synchrotron emissivity, i.e.

$$
Y\left(\gamma_{e}\right)=\frac{P_{s s c}\left(\gamma_{e}\right)}{P_{s y n}\left(\gamma_{e}\right)}
$$

When KN effects are unimportant, i.e. the IC scattering of $\gamma_{e}$ electrons with synchrotron photons are in the Thomson scattering regime, $Y\left(\gamma_{e}\right)$ is a constant and its value has been derived by Sari \& Esin (2001) for the GRB afterglow. However, for high energy electrons whose KN effect become important, $Y\left(\gamma_{e}\right)$ depends on $\gamma_{e}$. We approximate

$$
Y\left(\gamma_{e}\right)=\frac{U_{\text {syn }}\left[\nu<\nu_{K N}\left(\gamma_{e}\right)\right]}{U_{B}}
$$

for the afterglow emission (see also Li \& Waxman 2006), where $h \nu_{K N}\left(\gamma_{e}\right)=\Gamma m_{e} c^{2} / \gamma_{e}$ is the critic energy of scattering photons above which the scatterings with electrons of energy $\gamma_{e}$ just enter the KN scattering regime ( $\Gamma$ is the bulk Lorentz factor of the emission region), $U_{\text {syn }}\left[\nu<\nu_{K N}\left(\gamma_{e}\right)\right]$ is the energy density of the synchrotron photons with frequency below $\nu_{K N}\left(\gamma_{e}\right)$ and $U_{B}$ is the energy density of the magnetic field.

The KN effect can affect the electron distribution, which is given by

$$
N\left(\gamma_{e}\right)=\left\{\begin{array}{lr}
C_{1} \gamma_{e}^{-p} & \gamma_{m}<\gamma_{e}<\gamma_{c} \\
\frac{1+Y\left(\gamma_{c}\right)}{1+Y\left(\gamma_{e}\right)} C_{1} \gamma_{c} \gamma_{e}^{-p-1} & \gamma_{c}<\gamma_{e}
\end{array}\right.
$$

for the slow-cooling case and

$$
N\left(\gamma_{e}\right)=\frac{C_{2}}{1+Y\left(\gamma_{e}\right)}\left\{\begin{array}{lr}
\gamma_{e}^{-2} & \gamma_{c}<\gamma_{e}<\gamma_{m} \\
\gamma_{m}^{p-1} \gamma_{e}^{-p-1} & \gamma_{m}<\gamma_{e}
\end{array}\right.
$$

for the fast-cooling case respectively (Nakar et al. 2009), where $C_{1}$ and $C_{2}$ are two constants, $\gamma_{c}$ and $\gamma_{m}$ are cooling Lorentz factor and minimum Lorentz factor of electrons respectively (Sari et al. 1998). To compare with Fermi LAT observations, we consider the electrons that produce $h \nu_{*}=$ $100 \mathrm{MeV}$ synchrotron photons. We define $\gamma_{*}$ as the Lorentz factor of those electrons whose synchrotron frequency is $\nu_{*}$. The electrons producing such high-energy afterglow emission typically have $\gamma_{*} \gtrsim \max \left(\gamma_{c}, \gamma_{m}\right)$. For the slow-cooling case, one can derive the number density of electrons of $\gamma_{*}$ when the $\mathrm{KN}$ effect is taken into account, i.e.

$$
N\left(\gamma_{*}\right)=\frac{1+Y\left(\gamma_{c}\right)}{1+Y\left(\gamma_{*}\right)} C_{1} \gamma_{c} \gamma_{*}^{-p-1}=\frac{N_{s y n}\left(\gamma_{*}\right)}{1+Y\left(\gamma_{*}\right)},
$$

where $N_{\text {syn }}\left(\gamma_{*}\right)=C_{1} \gamma_{c, s y n} \gamma_{*}^{-p-1}=C_{1} \gamma_{c}\left[1+Y\left(\gamma_{c}\right)\right] \gamma_{*}^{-p-1}$ represents the number density of electrons of $\gamma_{*}$ when only the synchrotron cooling is considered $\left(\gamma_{c, s y n}\right.$ is the cooling Lorentz factor of electrons when only the synchrotron cooling is considered; see, e.g. Sari et al. 1998). Therefore the number density of electrons of $\gamma_{*}$ is a factor of $1+Y\left(\gamma_{*}\right)$ lower than that in the case that only the synchrotron cooling is considered. As a result, the synchrotron luminosity produced by $\gamma_{*}$ electrons is reduced by the same factor correspondingly.
In the fast-cooling case, when the IC scatterings of electrons of $\gamma_{e} \lesssim \gamma_{m}$ with synchrotron photons are in the Thomson scattering regime (the case of $\S 3.2 .2$ ), one can also obtain $N\left(\gamma_{*}\right)=N_{\text {syn }}\left(\gamma_{*}\right) /\left[1+Y\left(\gamma_{*}\right)\right]$, so the the synchrotron luminosity is also reduced by a factor of $1+Y\left(\gamma_{*}\right)$.

\section{KN EFFECT ON THE COMPTON PARAMETERS}

Now we derive $Y\left(\gamma_{*}\right)$. As the electron distribution is different in the fast and slow cooling cases, we divide the following analysis into these two different cases.

\subsection{The slow-cooling case}

Whether the afterglow emission belongs to the slowcooling or fast-cooling case depends on shock microphysics parameters (i.e. the magnetic field equipartition factor $\epsilon_{B}$ and electron energy equipartition factor $\epsilon_{e}$ ) and other parameters such as the burst energy $E$ and the circumburst density $n$. Among these parameters, the magnetic field equipartition factor $\epsilon_{B}$ is the mostly poorly known. The circumburst density $n$ depends on the burst environment and may range from $10^{-3} \mathrm{~cm}^{-3}$ to $10 \mathrm{~cm}^{-3}$ (e.g. Kumar \& Panaitescu 2001).

The condition for slow-cooling is

$$
n_{-1}^{1 / 2} \epsilon_{B,-5}<400\left[1+Y\left(\gamma_{c}\right)\right]^{-1} f_{p}^{-1} \epsilon_{e,-1}^{-1} E_{54}^{-1 / 2} t_{0}^{1 / 2}(1+z)^{-1 / 2},
$$

where $f_{p} \equiv 6(p-2) /(p-1), p$ is the power-law index of the electron energy distribution $(p=2.2$ has been used in the following calculations), $t_{0}$ is the time in units of $10^{0} \mathrm{~s}$ (hereafter we use the cgs units and denotation $Q_{x}=Q / 10^{x}$ ) and $z$ is the burst redshift. The cooling Lorentz factor and the minimum Lorentz factor of electrons in forward shocks are given by

$$
\gamma_{c}=10^{7}\left[1+Y\left(\gamma_{c}\right)\right]^{-1} E_{54}^{-3 / 8} n_{-1}^{-5 / 8} \epsilon_{B,-5}^{-1} t_{0}^{1 / 8}(1+z)^{-1 / 8}
$$

and

$$
\gamma_{m}=2.5 \times 10^{4} f_{p} \epsilon_{e,-1} E_{54}^{1 / 8} n_{-1}^{-1 / 8} t_{0}^{-3 / 8}(1+z)^{3 / 8},
$$

respectively, where $Y\left(\gamma_{c}\right)$ is the Compton parameter of the electrons of energy $\gamma_{c}$. The cooling frequency and minimum frequency of electrons corresponding to $\gamma_{c}$ and $\gamma_{m}$ are, respectively,

$$
\nu_{c}=8 \times 10^{22}\left[1+Y\left(\gamma_{c}\right)\right]^{-2} \epsilon_{B,-5}^{-3 / 2} E_{54}^{-1 / 2} n_{-1}^{-1} t_{0}^{-1 / 2}(1+z)^{-1 / 2} \mathrm{~Hz}
$$

and

$$
\nu_{m}=5 \times 10^{17} f_{p}^{2} \epsilon_{e,-1}^{2} \epsilon_{B,-5}^{1 / 2} E_{54}^{1 / 2} t_{0}^{-3 / 2}(1+z)^{1 / 2} \mathrm{~Hz} .
$$

In the slow-cooling case, the synchrotron luminosity is dominated by $\gamma_{c}$ electrons and the ratio of the SSC luminosity to synchrotron luminosity is approximately given by $Y\left(\gamma_{c}\right)$. Depending on the location of $\nu_{K N}\left(\gamma_{c}\right), U_{p h}\left[\nu<\nu_{K N}\left(\gamma_{c}\right)\right]$ is proportional to $\nu_{K N}\left(\gamma_{c}\right)^{(3-p) / 2}$ or $\nu_{K N}\left(\gamma_{c}\right)^{4 / 3}$. So the value of $Y\left(\gamma_{c}\right)$ can be obtained from

$$
\begin{aligned}
& Y\left(\gamma_{c}\right)\left[1+Y\left(\gamma_{c}\right)\right]=\frac{\epsilon_{e}}{\epsilon_{B}}\left(\frac{\gamma_{c}}{\gamma_{m}}\right)^{2-p} \\
& \times \begin{cases}\left(\frac{\nu_{m}}{\nu_{c}}\right)^{(3-p) / 2}\left(\frac{\nu_{K N}\left(\gamma_{c}\right)}{\nu_{m}}\right)^{4 / 3}, & \nu_{K N}\left(\gamma_{c}\right)<\nu_{m} \\
\left(\frac{\nu_{K N}\left(\gamma_{c}\right)}{\nu_{c}}\right)^{(3-p) / 2}, & \nu_{m}<\nu_{K N}\left(\gamma_{c}\right)<\nu_{c} \\
1 . & \nu_{c}<\nu_{K N}\left(\gamma_{c}\right)\end{cases}
\end{aligned}
$$

To calculate $Y\left(\gamma_{c}\right)$, we need to know the ratios of $\nu_{K N}\left(\gamma_{c}\right)$ to $\nu_{m}$ and $\nu_{c}$, which are respectively given by

$$
\frac{\nu_{K N}\left(\gamma_{c}\right)}{\nu_{c}}=7.5 \times 10^{-8}\left[1+Y\left(\gamma_{c}\right)\right]^{3} E_{54} \epsilon_{B,-5}^{5 / 2} n_{-1}^{3 / 2}(1+z)
$$


and

$$
\frac{\nu_{K N}\left(\gamma_{c}\right)}{\nu_{m}}=1.4 \times 10^{-2}\left[1+Y\left(\gamma_{c}\right)\right] f_{p}^{-2} \epsilon_{e,-1}^{-2} \epsilon_{B,-5}^{1 / 2} n_{-1}^{1 / 2} t_{0}
$$

Since we are interested in the high-energy afterglow emission, we also need to know the ratio of $\nu_{K N}\left(\gamma_{*}\right)$ to $\nu_{m}$, which is

$$
\begin{aligned}
& \frac{\nu_{K N}\left(\gamma_{*}\right)}{\nu_{m}}=\left(\frac{\nu_{c}}{\nu_{*}}\right)^{1 / 2} \frac{\nu_{K N}\left(\gamma_{c}\right)}{\nu_{m}} \\
& =2.8 \times 10^{-2} f_{p}^{-2} \epsilon_{e,-1}^{-2} \epsilon_{B,-5}^{-1 / 4} E_{54}^{-1 / 4} t_{0}^{3 / 4}(1+z)^{-1 / 4} .
\end{aligned}
$$

According to the relations among $\nu_{K N}\left(\gamma_{c}\right), \nu_{m}$ and $\nu_{c}$, we divide the discussion into three cases.

\subsubsection{Case I: $\nu_{K N}\left(\gamma_{c}\right)<\nu_{m}<\nu_{c}$}

This case typically happens at early times for reference parameter values we used. Eq.(11) can be simplified as

$$
\begin{aligned}
& Y\left(\gamma_{c}\right)\left[1+Y\left(\gamma_{c}\right)\right]=0.09\left[1+Y\left(\gamma_{c}\right)\right]^{7 / 3} \\
& \times f_{p}^{-5 / 3} \epsilon_{e,-1}^{-2 / 3} \epsilon_{B,-5}^{2 / 3} E_{54}^{1 / 2} n_{-1}^{7 / 6} t_{0}^{5 / 6}(1+z)^{1 / 2} .
\end{aligned}
$$

If $Y\left(\gamma_{c}\right) \ll 1$, one can obtain

$$
Y\left(\gamma_{c}\right)=0.09 f_{p}^{-5 / 3} \epsilon_{e,-1}^{-2 / 3} \epsilon_{B,-5}^{2 / 3} E_{54}^{1 / 2} n_{-1}^{7 / 6} t_{0}^{5 / 6}(1+z)^{1 / 2},
$$

while for $Y\left(\gamma_{c}\right) \gtrsim 1$, the value of $Y\left(\gamma_{c}\right)$ can be obtained only numerically.

One can also obtain the Compton parameter for those electrons that produce high-energy synchrotron emission with frequency $\nu_{*}$. Since usually $h \nu_{*}=100 \mathrm{MeV}>h \nu_{c}$, we only discuss the case of $\nu_{K N}\left(\gamma_{*}\right)<\nu_{K N}\left(\gamma_{c}\right)$ below ${ }^{7}$. For $\nu_{K N}\left(\gamma_{*}\right)<$ $\nu_{K N}\left(\gamma_{c}\right)<\nu_{m}$, we have

$$
\begin{aligned}
& Y\left(\gamma_{*}\right)=Y\left(\gamma_{c}\right)\left[\frac{\nu_{K N}\left(\gamma_{*}\right)}{\nu_{K N}\left(\gamma_{c}\right)}\right]^{4 / 3}=Y\left(\gamma_{c}\right)\left(\frac{\gamma_{*}}{\gamma_{c}}\right)^{-4 / 3}=Y\left(\gamma_{c}\right)\left(\frac{\nu_{*}}{\nu_{c}}\right)^{-2 / 3} \\
& =0.3 f_{p}^{-5 / 3} \epsilon_{e,-1}^{-2 / 3} \epsilon_{B,-5}^{-1 / 3} E_{54}^{1 / 6} n_{-1}^{1 / 2} t_{0}^{1 / 2}(1+z)^{1 / 6} .
\end{aligned}
$$

\subsubsection{Case II: $\nu_{m}<\nu_{K N}\left(\gamma_{c}\right)<\nu_{c}$}

As $\nu_{K N}\left(\gamma_{c}\right) / \nu_{m}$ increases with time, it is likely that $\nu_{K N}\left(\gamma_{c}\right)>\nu_{m}$ at later times. In this case, we have

$$
\begin{aligned}
& Y\left(\gamma_{c}\right)\left[1+Y\left(\gamma_{c}\right)\right]=\frac{\epsilon_{e}}{\epsilon_{B}}\left(\frac{\gamma_{c}}{\gamma_{m}}\right)^{2-p}\left(\frac{\nu_{K N}\left(\gamma_{c}\right)}{\nu_{c}}\right)^{(3-p) / 2} \\
& =1.2\left[1+Y\left(\gamma_{c}\right)\right]^{(5-p) / 2} f_{p}^{p-2} \epsilon_{e,-1}^{p-1} \epsilon_{B,-5}^{(3-p) / 4} E_{54}^{1 / 2} n_{-1}^{(5-p) / 4} t_{1}^{(2-p) / 2}(1+z)^{1 / 2} .
\end{aligned}
$$

Depending on whether $\nu_{K N}\left(\gamma_{*}\right)$ is larger or smaller than $\nu_{m}$, there are two sub-cases:

1) Case IIa: $\nu_{K N}\left(\gamma_{c}\right)>\nu_{m}>\nu_{K N}\left(\gamma_{*}\right)$. In this case,

$$
\begin{aligned}
& Y\left(\gamma_{*}\right)=Y\left(\gamma_{c}\right)\left[\frac{\nu_{m}}{\nu_{K N}\left(\gamma_{c}\right)}\right]^{(3-p) / 2}\left[\frac{\nu_{K N}\left(\gamma_{*}\right)}{\nu_{m}}\right]^{4 / 3} \\
& =0.9 f_{p}^{-5 / 3} \epsilon_{e,-1}^{-2 / 3} \epsilon_{B,-5}^{-1 / 3} E_{54}^{1 / 6} n_{-1}^{1 / 2} t_{1}^{1 / 2}(1+z)^{1 / 6} .
\end{aligned}
$$

2) Case IIb: $\nu_{K N}\left(\gamma_{c}\right)>\nu_{K N}\left(\gamma_{*}\right)>\nu_{m}$. In this case,

$$
\begin{aligned}
& Y\left(\gamma_{*}\right)=Y\left(\gamma_{c}\right)\left[\frac{\nu_{K N}\left(\gamma_{*}\right)}{\nu_{K}\left(\gamma_{)}\right)}\right]^{(3-p) / 2}=Y\left(\gamma_{c}\right)\left(\frac{\nu_{*}}{\nu_{c}}\right)^{(p-3) / 4} \\
& =2 f_{p}^{p-2} \epsilon_{e,-1}^{p-1} \epsilon_{B,-5}^{(p-3) / 8} E_{54}^{(p+1) / 8} n_{-1}^{1 / 2} t_{2}^{(5-3 p) / 8}(1+z)^{(p-2) / 2} .
\end{aligned}
$$

\footnotetext{
${ }^{7}$ When the number density $n$ is very low (e.g. $n \lesssim 10^{-2} \mathrm{~cm}^{-2}$ ), $h \nu_{*}$ can be lower than $h \nu_{c}$ and $\nu_{K N}\left(\gamma_{*}\right)>\nu_{K N}\left(\gamma_{c}\right) . Y\left(\gamma_{*}\right)$ can be similarly obtained in this case. In figures 1 and 2 where $Y\left(\gamma_{*}\right)$ is calculated for $n$ ranging from $10^{-3}$ to $10 \mathrm{~cm}^{-3}$ and $\epsilon_{B}$ ranging from $10^{-1}$ to $10^{-6}$, this situation has been included in the calculation.
}

3.1.3. Case III: $\nu_{K N}\left(\gamma_{c}\right)>\nu_{c}>\nu_{m}$

In this case, the $\mathrm{KN}$ effect on $\gamma_{c}$ electrons is not important and the Compton parameter is given by

$$
\begin{aligned}
& Y\left(\gamma_{c}\right)\left[1+Y\left(\gamma_{c}\right)\right]=\frac{\epsilon_{e}}{\epsilon_{B}}\left(\frac{\gamma_{c}}{\gamma_{m}}\right)^{2-p}=2.4 \times 10^{3}\left[1+Y\left(\gamma_{c}\right)\right]^{p-2} f_{p}^{p-2} \\
& \times \epsilon_{e,-1}^{p-1} \epsilon_{B,-5}^{p-3} E_{54}^{(p-2) / 2} n_{-1}^{(p-2) / 2} t_{1}^{-(p-2) / 2}(1+z)^{(p-2) / 2} .
\end{aligned}
$$

Depending on the relations among $\nu_{K N}\left(\gamma_{*}\right), \nu_{m}$ and $\nu_{c}$, there are three sub-cases:

1) Case III a: $\nu_{K N}\left(\gamma_{*}\right)<\nu_{m}<\nu_{c}<\nu_{K N}\left(\gamma_{c}\right)$. Define $\hat{\gamma}_{c}=$ $\Gamma m_{e} c^{2} / \nu_{c}$ as the critic Lorentz factor of those electrons that their interaction with synchrotron peak photons (i.e. photons at $\nu_{c}$ ) is just in the $\mathrm{KN}$ regime. In this case, we have

$$
\begin{aligned}
& Y\left(\gamma_{*}\right)=Y\left(\hat{\gamma}_{c}\right)\left(\frac{\nu_{m}}{\nu_{c}}\right)^{(3-p) / 2}\left[\frac{\nu_{K N}\left(\gamma_{*}\right)}{\nu_{m}}\right]^{4 / 3} \\
& =0.9 f_{p}^{-5 / 3} \epsilon_{e,-1}^{-2 / 3} \epsilon_{B,-5}^{-1 / 3} E_{54}^{1 / 6} n_{-1}^{1 / 2} t_{1}^{1 / 2}(1+z)^{1 / 6},
\end{aligned}
$$

where $Y\left(\hat{\gamma}_{c}\right)=Y\left(\gamma_{c}\right)$ has been used in the last step.

2) Case IIIb: $\nu_{m}<\nu_{K N}\left(\gamma_{*}\right)<\nu_{c}<\nu_{K N}\left(\gamma_{c}\right)$. In this case,

$$
\begin{aligned}
& Y\left(\gamma_{*}\right)=Y\left(\hat{\gamma}_{c}\right)\left[\frac{\nu_{K N}\left(\gamma_{*}\right)}{\nu_{c}}\right]^{(3-p) / 2}=Y\left(\gamma_{c}\right)\left[\frac{\nu_{*}}{\nu_{c}}{ }^{(p-3) / 4}\left(\frac{\nu_{K N}\left(\gamma_{c}\right)}{\nu_{c}}\right)^{(3-p) / 2}\right. \\
& =2 f_{p}^{p-2} \epsilon_{e,-1}^{p-1} \epsilon_{B,-5}^{(p-3) / 8} E_{54}^{(p+1) / 8} n_{-1}^{1 / 2} t_{2}^{(5-3 p) / 8}(1+z)^{(p-2) / 2} .
\end{aligned}
$$

3) Case IIIc: $\nu_{m}<\nu_{c}<\nu_{K N}\left(\gamma_{*}\right)<\nu_{K N}\left(\gamma_{c}\right)$. In this case,

$$
Y\left(\gamma_{*}\right)=Y\left(\gamma_{c}\right) .
$$

\subsection{The fast-cooling case}

The condition for the fast-cooling case is

$$
n_{-1}^{1 / 2} \epsilon_{B,-2} \gtrsim 0.4\left[1+Y\left(\gamma_{c}\right)\right]^{-1} f_{p}^{-1} \epsilon_{e,-1}^{-1} E_{54}^{-1 / 2} t_{0}^{1 / 2}(1+z)^{-1 / 2} .
$$

Below we use a larger $\epsilon_{B}$ as the reference value for the fastcooling case. In this case, the synchrotron radiation is dominated by $\gamma_{m}$ electrons and the critic frequency of interest is

$$
\nu_{K N}\left(\gamma_{m}\right)=3.7 \times 10^{18} f_{p}^{-1} \epsilon_{e,-1}^{-1} \mathrm{~Hz} .
$$

Similarly, one can find the ratios of $\nu_{K N}\left(\gamma_{m}\right)$ to two characteristic frequencies,

$$
\begin{gathered}
\frac{\nu_{K N}\left(\gamma_{m}\right)}{\nu_{m}}=0.24 f_{p}^{-3} \epsilon_{e,-1}^{-3} E_{54}^{-1 / 2} \epsilon_{B,-2}^{-1 / 2} t_{0}^{3 / 2}(1+z)^{-1 / 2} \\
\frac{\nu_{K N}\left(\gamma_{m}\right)}{\nu_{c}}=1.5\left[1+Y\left(\gamma_{c}\right)\right]^{2} f_{p}^{-1} \epsilon_{e,-1}^{-1} E_{54}^{1 / 2} \epsilon_{B,-2}^{3 / 2} n_{-1} t_{0}^{1 / 2}(1+z)^{1 / 2} .
\end{gathered}
$$

Below we divide the discussion into two cases according to whether the $\mathrm{KN}$ effect of $\gamma_{m}$ electrons is important or not, i.e. the cases of $\nu_{K N}\left(\gamma_{m}\right)<\nu_{m}$ and $\nu_{K N}\left(\gamma_{m}\right)>\nu_{m}$.

\subsubsection{Case I: $\nu_{K N}\left(\gamma_{m}\right)<\nu_{m}$}

Different from the slow-cooling case, the electron distribution at low-energies is affected by the $\mathrm{KN}$ effect in this case and therefore the corresponding synchrotron spectrum may be changed. Following Nakar et al. (2009), we define $\nu_{0}$ as the synchrotron frequency of electrons of $\gamma_{0}$ (i.e. $\left.\nu_{0}=\nu_{\text {syn }}\left(\gamma_{0}\right)\right)$, where $Y\left(\gamma_{0}\right)=1$. According to whether $\gamma_{m}$ is greater or smaller than $\gamma_{0}$, there are two subcases, i.e. 1) $\gamma_{0}<\gamma_{m}$ and 2) $\gamma_{0}>\gamma_{m}$.

i)Case Ia: $\gamma_{0}<\gamma_{m}$. This case applies when $\epsilon_{B}$ is large. Define $\hat{\gamma}_{0}=\Gamma m_{e} c^{2} / h \nu_{0}$ and $\hat{\gamma}_{m}=\Gamma m_{e} c^{2} / h \nu_{m}$. In the energy range $\hat{\gamma}_{0}<\gamma_{e}<\gamma_{0}$, the electron distribution is $N\left(\gamma_{e}\right) \propto \gamma_{e}^{-1}$ 
and the synchrotron spectrum is $\nu F_{\nu} \propto \nu$ (Wang et al. 2009; Nakar et al. 2009). Hence we have $Y\left(\gamma_{0}\right)=Y\left(\hat{\gamma}_{0}\right)\left(\gamma_{0} / \hat{\gamma}_{0}\right)^{-1}$. Since $\nu F_{\nu}\left(\nu_{0}\right)=\nu F_{\nu}\left(\nu_{m}\right)\left(\nu_{0} / \nu_{m}\right)^{1 / 2}$ and $Y\left(\hat{\gamma}_{m}\right)=\epsilon_{e} / \epsilon_{B}$ in this case, $Y\left(\hat{\gamma}_{0}\right)=\frac{\epsilon_{e}}{\epsilon_{B}}\left(\frac{\nu_{0}}{\nu_{m}}\right)^{1 / 2}$. Then we obtain $\gamma_{0}=\hat{\gamma}_{0}\left(\frac{\epsilon_{e}}{\epsilon_{B}}\right)\left(\frac{\nu_{0}}{\nu_{m}}\right)^{1 / 2}$. From

$$
\frac{\nu_{0}}{\nu_{m}}=\left(\frac{\gamma_{0}}{\gamma_{m}}\right)^{2}=0.06 f_{p}^{-3} \epsilon_{e,-1}^{-2} E_{54}^{-1 / 2} \epsilon_{B,-1}^{-3 / 2} t_{0}^{3 / 2}(1+z)^{-1 / 2},
$$

one can further obtain the corresponding synchrotron frequency of $\gamma_{0}$ electrons,

$$
\nu_{0}=3.6 \times 10^{18} f_{p}^{-1} \epsilon_{B,-1}^{-1} \mathrm{~Hz} .
$$

Similarly, one can obtain the synchrotron frequency of $\hat{\gamma}_{0}$ electrons,

$$
\begin{aligned}
& \hat{\nu}_{0}=\left(\frac{\hat{\gamma}_{0}}{\gamma_{m}}\right)^{2} \nu_{m}=\left[\frac{\nu_{K N}\left(\gamma_{m}\right)}{\nu_{0}}\right]^{2} \nu_{m} \\
& =5 \times 10^{19} f_{p}^{2} \epsilon_{B,-1}^{5 / 2} E_{54}^{1 / 2} t_{0}^{-3 / 2}(1+z)^{1 / 2} \mathrm{~Hz} .
\end{aligned}
$$

If $\max \left(\nu_{c}, \hat{\nu}_{0}\right) \lesssim \nu_{K N}\left(\gamma_{m}\right) \lesssim \nu_{0} \lesssim \nu_{m}$, we have

$$
Y\left(\gamma_{m}\right)\left[1+Y\left(\gamma_{m}\right)\right]=\frac{\epsilon_{e}}{\epsilon_{B}}\left(\frac{\nu_{K N}\left(\gamma_{m}\right)}{\nu_{0}}\right)\left(\frac{\nu_{0}}{\nu_{m}}\right)^{1 / 2} .
$$

As $Y\left(\gamma_{m}\right)<1$ when $\gamma_{0}<\gamma_{m}$, we obtain

$$
Y\left(\gamma_{m}\right) \simeq \frac{\epsilon_{e}}{\epsilon_{B}}\left(\frac{\nu_{K N}\left(\gamma_{m}\right)}{\nu_{0}}\right)\left(\frac{\nu_{0}}{\nu_{m}}\right)^{1 / 2}=0.2 f_{p}^{-3 / 2} \epsilon_{e,-1}^{-1} E_{54}^{-1 / 4} \epsilon_{B,-1}^{-3 / 4} t_{0}^{3 / 4}(1+z)^{-1 / 4} .
$$

If $\max \left(\nu_{c}, \hat{\nu}_{0}\right) \lesssim \nu_{K N}\left(\gamma_{*}\right) \lesssim \nu_{K N}\left(\gamma_{m}\right) \lesssim \nu_{0}$, it is easy to obtain

$$
Y\left(\gamma_{*}\right)=Y\left(\gamma_{m}\right) \frac{\nu_{K N}\left(\gamma_{*}\right)}{\nu_{K N}\left(\gamma_{m}\right)}=Y\left(\gamma_{m}\right)\left(\frac{\nu_{*}}{\nu_{m}}\right)^{-1 / 2}=0.01 f_{p}^{-1 / 2} \epsilon_{B,-1}^{-1 / 2} .
$$

In other cases, the derivation of $Y\left(\gamma_{*}\right)$ is complicated. However, we note that as long as $\nu_{*}>\nu_{0}=3.6 \times 10^{18} f_{p}^{-1} \epsilon_{B,-1}^{-1} \mathrm{~Hz}$,

$$
Y\left(\gamma_{*}\right)<1
$$

For $h \nu_{*}=100 \mathrm{MeV}, \nu_{*}>\nu_{0}$ is satisfied given that the fastcooling condition is satisfied. Therefore we conclude that $Y\left(\gamma_{*}\right)<1$ in case Ia.

ii) Case Ib: $\gamma_{0}>\gamma_{m}$. This case applies when $\epsilon_{B}$ is smaller. In this case, $\nu_{K N}\left(\gamma_{m}\right) \lesssim \nu_{m} \lesssim \nu_{0}$, so

$$
Y\left(\gamma_{m}\right)\left[1+Y\left(\gamma_{m}\right)\right]=\frac{\epsilon_{e}}{\epsilon_{B}} \frac{\nu_{K N}\left(\gamma_{m}\right)}{\nu_{m}} .
$$

From $Y\left(\gamma_{0}\right)=Y\left(\gamma_{m}\right)\left(\frac{\gamma_{0}}{\gamma_{m}}\right)^{-1}=1$, we obtain $\gamma_{0}=\frac{\epsilon_{e}}{\epsilon_{B}} \frac{\nu_{K N}\left(\gamma_{m}\right)}{\nu_{m}} \gamma_{m}$ and

$$
\nu_{0}=3.6 \times 10^{19} f_{p}^{-1} \epsilon_{B,-2}^{-1} \mathrm{~Hz} .
$$

As, $Y\left(\gamma_{m}\right)>1$ in this case, we get

$$
Y\left(\gamma_{m}\right)=\left[\frac{\epsilon_{e}}{\epsilon_{B}} \frac{\nu_{K N}\left(\gamma_{m}\right)}{\nu_{m}}\right]^{1 / 2}=1.2 f_{p}^{-3 / 2} \epsilon_{e,-1}^{-1} E_{54}^{-1 / 4} \epsilon_{B,-2}^{-3 / 4} t_{0}^{3 / 4}(1+z)^{-1 / 4}
$$

Similarly, if $\max \left(\nu_{c}, \hat{\nu}_{0}\right) \lesssim \nu_{K N}\left(\gamma_{*}\right) \lesssim \nu_{K N}\left(\gamma_{m}\right) \lesssim \nu_{0}$

$$
Y\left(\gamma_{*}\right)=Y\left(\gamma_{m}\right) \frac{\nu_{K N}\left(\gamma_{*}\right)}{\nu_{K N}\left(\gamma_{m}\right)}=0.03 f_{p}^{-1 / 2} \epsilon_{B,-2}^{-1 / 2}
$$

In other case, we also have

$$
Y\left(\gamma_{*}\right)<1
$$

as long as $\nu_{*}>\nu_{0}$ is satisfied.

\subsubsection{Case II: $\nu_{K N}\left(\gamma_{m}\right)>\nu_{m}$}

At later times, when $t>3 f_{p}^{2} \epsilon_{e,-1}^{2} E_{54}^{1 / 3} \epsilon_{B,-2}^{1 / 3}(1+z)^{1 / 3} \mathrm{~s}$, $\nu_{K N}\left(\gamma_{m}\right)>\nu_{m}$. In this case, the KN effect of $\gamma_{m}$ electrons is unimportant and

$$
Y\left(\gamma_{m}\right)=\left(\frac{\epsilon_{e}}{\epsilon_{B}}\right)^{1 / 2}=3 \epsilon_{e,-1}^{1 / 2} \epsilon_{B,-2}^{-1 / 2},
$$

as the synchrotron emission typically peaks at $\nu_{m}$ in this case (Nakar et al. 2009). In order to calculate $Y\left(\gamma_{*}\right)$, let's first derive the ratios of $\nu_{K N}\left(\gamma_{*}\right)$ to two critic frequencies, which are respectively

$$
\begin{aligned}
& \frac{\nu_{K N}\left(\gamma_{*}\right)}{\nu_{m}}=\left(\frac{\nu_{m}}{\nu_{*}}\right)^{1 / 2} \frac{\nu_{K N}\left(\gamma_{m}\right)}{\nu_{m}} \\
& =0.04 f_{p}^{-2} \epsilon_{e,-1}^{-2} \epsilon_{B,-2}^{-1 / 4} E_{54}^{-1 / 4} t_{1}^{3 / 4}(1+z)^{-1 / 4}
\end{aligned}
$$

and

$$
\begin{aligned}
& \frac{\nu_{K N}\left(\gamma_{*}\right)}{\nu_{c}}=\left(\frac{\nu_{m}}{\nu_{*}}\right)^{1 / 2} \frac{\nu_{K N}\left(\gamma_{m}\right)}{\nu_{c}} \\
& =0.25 \epsilon_{e,-1} \epsilon_{B,-2}^{3 / 4} E_{54}^{3 / 4} n_{-1} t_{1}^{-1 / 4}(1+z)^{3 / 4},
\end{aligned}
$$

where $Y\left(\gamma_{c}\right)=Y\left(\gamma_{m}\right)=\sqrt{\epsilon_{e} / \epsilon_{B}}$ has been used. According to the relations among $\nu_{K N}\left(\gamma_{*}\right), \nu_{m}$ and $\nu_{c}$, there are three subcases:

1)Case IIa: $\nu_{c}<\nu_{K N}\left(\gamma_{*}\right)<\nu_{m}$. In this case,

$Y\left(\gamma_{*}\right)=Y\left(\hat{\gamma}_{m}\right)\left(\frac{\nu_{K N}\left(\gamma_{*}\right)}{\nu_{m}}\right)^{1 / 2}=0.7 f_{p}^{-1} \epsilon_{e,-1}^{-1 / 2} \epsilon_{B,-2}^{-5 / 8} E_{54}^{-1 / 8} t_{1}^{3 / 8}(1+z)^{-1 / 8}$,

where $\hat{\gamma}_{m}=\Gamma m_{e} c^{2} / h \nu_{m}$ is the critic Lorentz factor of those electrons that their interaction with photons at $\nu_{m}$ is just in the KN regime and $Y\left(\hat{\gamma}_{m}\right)=Y\left(\gamma_{m}\right)=\sqrt{\epsilon_{e} / \epsilon_{B}}$ has been used in the last step.

2)Case IIb: $\nu_{K N}\left(\gamma_{*}\right)<\nu_{c}$. In this case,

$$
\begin{aligned}
& Y\left(\gamma_{*}\right)=Y\left(\hat{\gamma}_{m}\right)\left(\frac{\nu_{c}}{\nu_{m}}\right)^{1 / 2}\left(\frac{\nu_{K N}\left(\gamma_{*}\right)}{\nu_{c}}\right)^{4 / 3}=2.2\left[1+Y\left(\gamma_{c}\right)\right]^{-1} f_{p}^{-1} \epsilon_{e,-1}^{5 / 6} \\
& \times \epsilon_{B,-2}^{-1 / 2} E_{54}^{1 / 2} n_{-1}^{5 / 6} t_{1}^{1 / 6}(1+z)^{1 / 2}=0.18 f_{p}^{-1} \epsilon_{e,-1}^{1 / 3} E_{54}^{1 / 2} n_{-1}^{5 / 6} t_{1}^{1 / 6}(1+z)^{1 / 2},
\end{aligned}
$$

3)Case IIc: $\nu_{K N}\left(\gamma_{*}\right)>\nu_{m}$. In this case,

$$
Y\left(\gamma_{*}\right)=Y\left(\gamma_{m}\right)=3 \epsilon_{e,-1}^{1 / 2} \epsilon_{B,-2}^{-1 / 2} .
$$

\section{KN EFFECT ON THE HIGH-ENERGY SYNCHROTRON AFTERGLOW LUMINOSITY}

The above analyses give the dependence of the Compton parameters $Y\left(\gamma_{*}\right), Y\left(\gamma_{c}\right)$ (in the slow-cooling case) and $Y\left(\gamma_{m}\right)$ (in the fast-cooling case) on the parameters such as $\epsilon_{e}, \epsilon_{B}, E$ and $n$. Since $\epsilon_{B}$ and $n$ are the least known among these parameters for GRB afterglows, we explore the value of $Y\left(\gamma_{*}\right)$ and $Y\left(\gamma_{c}\right)$ (or $\left.Y\left(\gamma_{m}\right)\right)$ as a function of these two parameters. In Figures 1 and 2, we show the result for two different times, i.e. at $t=1 \mathrm{~s}$ and $t=10 \mathrm{~s}$ respectively. We find $Y\left(\gamma_{*}\right)$ is smaller than a few at $t=1 \mathrm{~s}$ for the parameters $\epsilon_{B}$ in the range from $10^{-6}$ to $10^{-1}$ and $n$ in the range from $10^{-3} \mathrm{~cm}^{-3}$ to $10 \mathrm{~cm}^{-3}$. At $t=10 \mathrm{~s}$, $Y\left(\gamma_{*}\right)$ is also smaller than a few in a wide range of parameter space (it is large than a few only when $n$ is as high as $10 \mathrm{~cm}^{-3}$ and $\epsilon_{B}$ is close to $\left.10^{-6}\right)$. On the other hand, $Y\left(\gamma_{c}\right)$ or $Y\left(\gamma_{m}\right)$ can be more than one order of magnitude higher in the same parameter space. This implies that SSC loss of high-energy electrons that produce high-energy ( $\gtrsim 100 \mathrm{MeV}$ ) afterglow photons is typically small. As a result, the synchrotron luminosity at high-energies is correspondingly high, which enables the detection of early high-energy afterglow emission by Fermi LAT. 

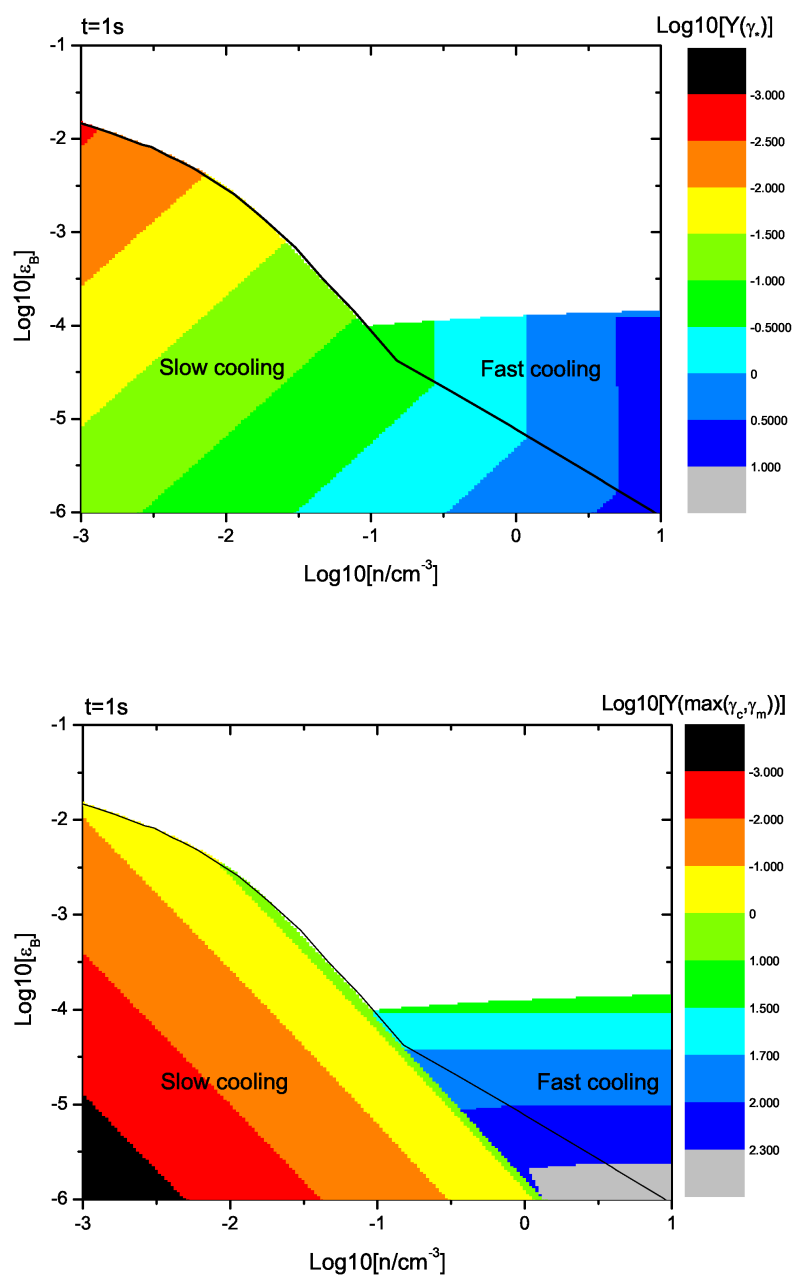

FIG. 1.- Values of the Compton parameters $Y\left(\gamma_{*}\right)$ (top panel) and $Y\left(\gamma_{c}\right)$ (or $Y\left(\gamma_{m}\right)$, bottom panel) of the afterglow emission as a function of $\epsilon_{B}$ and $n$ at time $t=1 \mathrm{~s}$ after the burst. The black solid line separates the slow-cooling case and fast-cooling case. The blank space in the plot corresponds to the region where $\nu_{K N}\left(\gamma_{m}\right)<\nu_{m}$, for which $Y\left(\gamma_{*}\right)<1$ but the exact value is not calculated. Other parameters used in the plots are $\epsilon_{e}=0.1$ and $E=10^{54} \mathrm{erg}$.

In order to see whether the SSC emission contributes to the high-energy afterglow emission at Fermi LAT energy band, we calculate the spectral energy distribution of the afterglow emission at early times numerically. Assuming an adiabatic evolution of the blast wave and using the electron distribution given in $\S 2$, we calculate the synchrotron radiation spectrum as well as the SSC spectrum with a full $\mathrm{KN}$ cross section taken into account (see equations 2 and 11 of He et al. (2009) for the description of the dynamic and the full $\mathrm{KN}$ cross section). Figure 3 shows the $\nu F_{\nu}$ spectra of the afterglow synchrotron emission and the SSC emission for the slow-cooling case at times $t=1 \mathrm{~s}$ and $t=10 \mathrm{~s}$ when the $\mathrm{KN}$ effect is taken into account. In the Fermi LAT energy band, the synchrotron component is dominated at both times. The SSC component becomes dominated only at energies above the maximum synchrotron photon energy of shock-accelerated electrons, at which the flux usually becomes, however, too low to be detectable by Fermi LAT. The spectral energy distribution of the afterglow emission for the fast-cooling case at times $t=10 \mathrm{~s}$ and $t=100 \mathrm{~s}$ is shown in Fig.4. Similarly, SSC contribution to
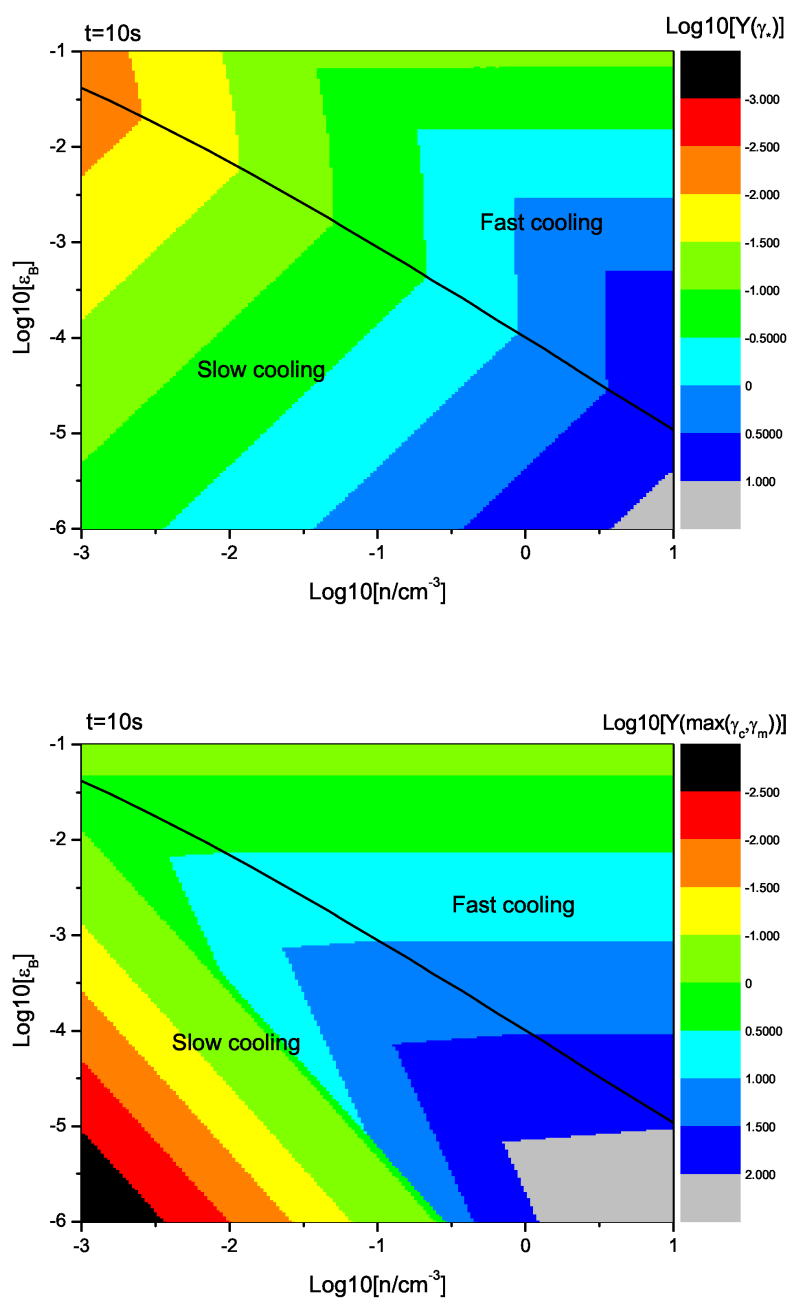

FIG. 2.- The same as figure 1, but at time $t=10 \mathrm{~s}$ after the burst.

the high-energy emission at energies below $100 \mathrm{GeV}$ is negligible at these times. Fig.4 (see the bottom panel) also shows that the spectrum becomes harder at energies above $10^{7} \mathrm{eV}$. This is caused by the decreased IC loss suppression on the synchrotron flux at high energies due to the KN effect.

The Compton parameters also vary with time. In the above analytic calculation in $\S 3$, we have shown that, $Y\left(\gamma_{*}\right)$ increases with time as $t^{1 / 2}$ in the slow-cooling case as long as $\nu_{K N}\left(\gamma_{*}\right)<\nu_{m}$. When $\nu_{K N}\left(\gamma_{*}\right)>\nu_{m}, Y\left(\gamma_{*}\right)$ starts to decrease with time. We calculate $Y\left(\gamma_{c}\right)$ numerically using Eq.11 and show the evolution of $Y\left(\gamma_{c}\right)$ and $Y\left(\gamma_{*}\right)$ with time in the top panel of Fig.5. If $Y\left(\gamma_{*}\right) \gtrsim 1$ as well, as in the case of some parameter space shown in figures 1 and 2, the decay of the synchrotron afterglow emission will be faster than what is predicted by the standard synchrotron afterglow theory (i.e. steeper than $t^{(2-3 p) / 4}$ for $\left.\nu_{*}>\nu_{c}\right)$, since the synchrotron luminosity at frequency $\nu_{*}$ scales as $1 /\left[1+Y\left(\gamma_{*}\right)\right]$. The decay could be steeper by a factor $\Delta \alpha=1 / 2$ at most. We numerically calculate the light curves of the afterglow emission assuming an adiabatic evolution of the blast wave and using the electron distribution given in $\S 2$. The light curves of the synchrotron emission, the SSC emission and the sum of them at frequency $\nu_{*}=100 \mathrm{MeV}$ are shown in the bottom panel of fig- 


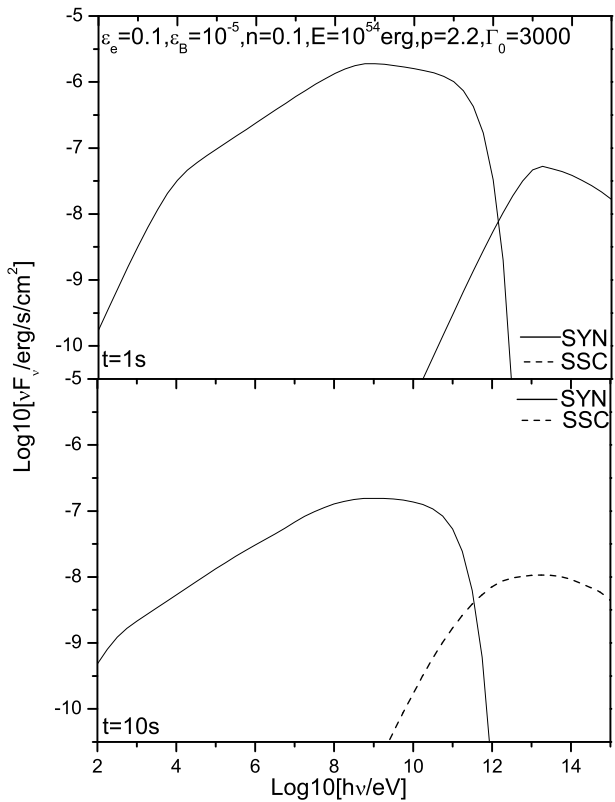

FIG. 3.- The calculated spectral energy distribution of the afterglow emission in the slow-cooling case at times $t=1 \mathrm{~s}$ and $t=10 \mathrm{~s}$ after the burst with the $\mathrm{KN}$ effect taken into account. The solid and dashed lines represent the synchrotron component and SSC component, respectively.

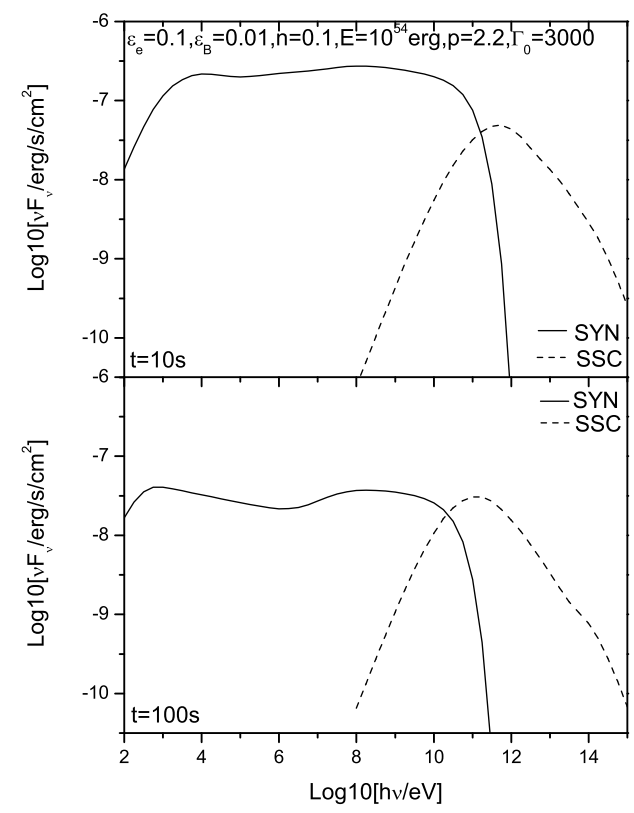

FIG. 4.- The calculated spectral energy distribution of the afterglow emission in the fast-cooling case at times $t=10 \mathrm{~s}$ and $t=100 \mathrm{~s}$ after the burst with the KN effect taken into account. The solid and dashed lines represent the synchrotron component and SSC component, respectively.

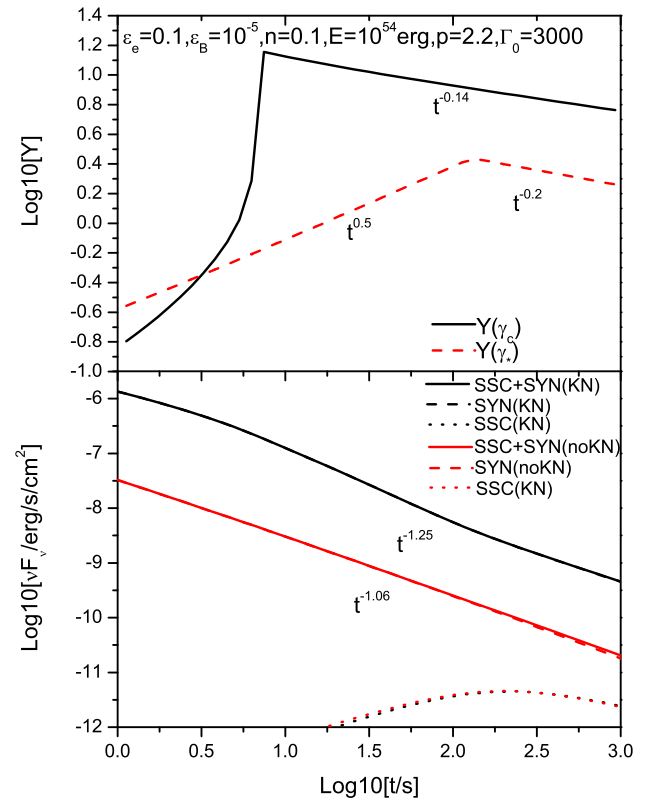

FIG. 5.- The top panel shows the evolution of Compton parameters $Y\left(\gamma_{*}\right)$ and $Y\left(\gamma_{c}\right)$ with time in the slow-cooling case for the parameters $\epsilon_{e}=0.1$, $\epsilon_{B}=10^{-5}, n=0.1 \mathrm{~cm}^{-3}, E=10^{54} \mathrm{ergs}, p=2.2$ and $\Gamma_{0}=3000$. The bottom panel shows the light curves of the synchrotron emission, the SSC emission and the sum of them at $h \nu_{*}=100 \mathrm{MeV}$. Black lines and red lines denote, respectively, the light curves with and without $\mathrm{KN}$ effect taken into account.

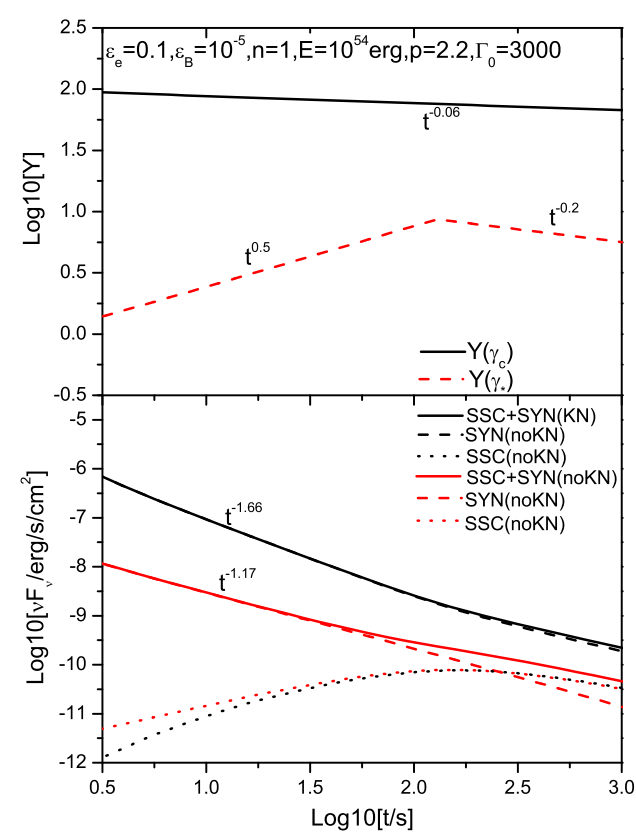

FIG. 6.- The same as figure 5, but a larger circumburst density of $n=$ $1 \mathrm{~cm}^{-3}$. 


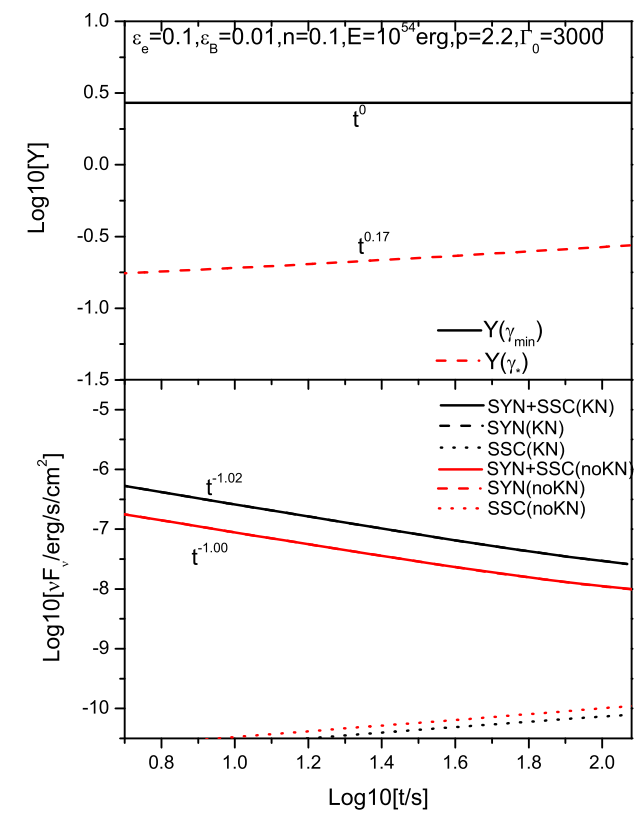

FIG. 7. - The top panel shows the evolution of Compton parameters $Y\left(\gamma_{*}\right)$ and $Y\left(\gamma_{m}\right)$ with time in the fast-cooling case for the parameters $\epsilon_{e}=0.1$, $\epsilon_{B}=0.01, n=0.1 \mathrm{~cm}^{-3}, E=10^{54} \mathrm{ergs}, p=2.2$ and $\Gamma_{0}=3000$. The bottom panel shows the light curves of the synchrotron emission, the SSC emission and the sum of them at $h \nu_{*}=100 \mathrm{MeV}$. Black lines and red lines denote, respectively, the light curves with and without $\mathrm{KN}$ effect taken into account.

ure 5 (the black lines). As a comparison, we also show the light curves (the red lines) in the case that the $\mathrm{KN}$ effect is not taken into account (i.e. assuming that the SSC cooling is in the Thomson regime). One can see that the light curve decay becomes steeper and the flux at $100 \mathrm{MeV}$ is significantly higher when the KN effect is taken into account. In Fig.6, we also show the light curves for another set of parameters in the slowcooling case. It also shows that the temporal decay of highenergy afterglow emission can be significantly steeper than what is predicted by the standard synchrotron theory when the $\mathrm{KN}$ effect is taken into account.

For the fast-cooling case, in the above analytic calculations we have found that when $\nu_{K N}\left(\gamma_{m}\right)<\nu_{m}, \nu_{*}$ is always larger than $\nu_{0}$ (except when $\epsilon_{B}<10^{-5}$, but note that for $\epsilon_{B}<10^{-5}$, the afterglow emission is no longer in the fast-cooling case and the discussion in $\S 3.1$ applies). For $\nu_{*}>\nu_{0}, Y\left(\gamma_{*}\right)<1$, so almost all of the energy of high-energy electrons goes into the synchrotron emission and the synchrotron luminosity is high. As $Y\left(\gamma_{*}\right)<1$, the decay slope of the high-energy emission will not be affected. However, in the case of $\nu_{K N}\left(\gamma_{m}\right)>\nu_{m}$, $Y\left(\gamma_{*}\right)$ could be larger than 1 and it increases with time as $t^{3 / 8}$ or $t^{1 / 6}$ in some certain parameter space. This will lead to a steeper decay of the synchrotron afterglow emission at frequency $\nu_{*}$. Figures 7 and 8 show the time evolution of the Compton parameters $Y\left(\gamma_{*}\right)$ and $Y\left(\gamma_{m}\right)$, and the light curves of the high-energy afterglow emission at $\nu_{*}=100 \mathrm{MeV}$ for two set of parameters. They clearly indicate that the temporal decay of high-energy afterglow emission becomes steeper than $t^{(2-3 p) / 4}$ when $Y\left(\gamma_{*}\right) \gtrsim 1$. So we conclude that the light curve of the high-energy afterglow emission could be also steeper in the fast-cooling case when the $\mathrm{KN}$ effect is taken into account.

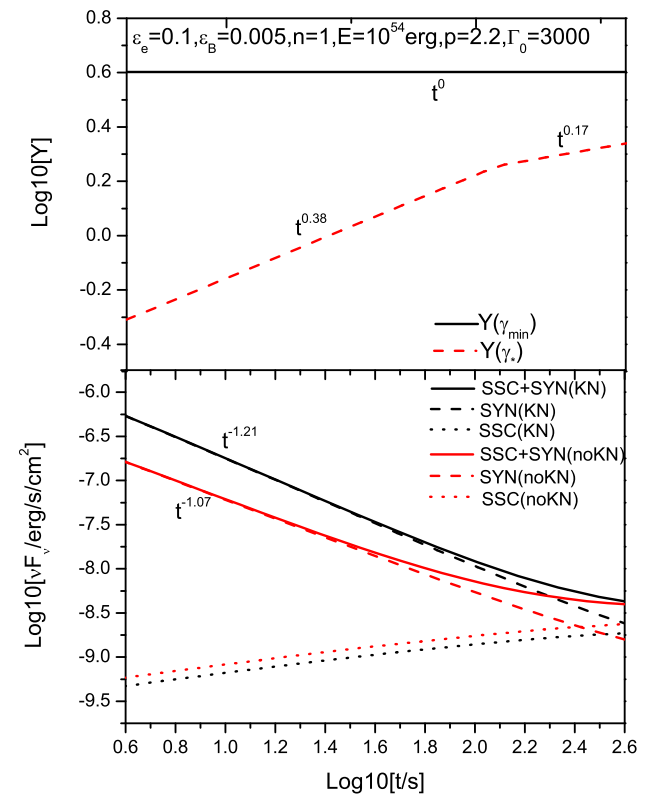

FIG. 8. - The same as figure 7, but for $\epsilon_{B}=0.005$ and $n=1 \mathrm{~cm}^{-3}$.

\section{IMPLICATION FOR FERMI LAT OBSERVATION OF AFTERGLOW} EMISSION

As has been shown in figures 1 and 2, the Compton parameters for the electrons that produce high-energy gamma-ray afterglow emission are typically small at early time $t \lesssim 10$ s, i.e. $Y\left(\gamma_{*}\right) \lesssim$ a few for a wide range of parameter space. This has important implication for the detectability of high-energy afterglow emission by Fermi LAT, since a low $Y\left(\gamma_{*}\right)$ leads to a high synchrotron luminosity at high-energies. The lowenergy electrons that produce early x-ray and optical afterglow emission, however, still suffers from the strong IC loss (i.e. $Y\left(\gamma_{c}\right)$ or $Y\left(\gamma_{m}\right)$ are typically high) and therefore the observed early $\mathrm{x}$-ray/optical afterglow luminosity is low, compared with high-energy gamma-ray emission.

The time evolution of $Y\left(\gamma_{*}\right)$ also has implication for the temporal decay slope of high-energy afterglow emission. $Y\left(\gamma_{*}\right)$ increases with time and its value could be greater than 1 at late times for some range of parameter space. This will lead to a faster decay of the high-energy synchrotron afterglow emission, which may explain the early fast decay of the high-energy gamma-ray emission seen in GRB090510 and GRB090902B.

The Fermi LAT and GBM observations as well as the Swift observations of the short burst GRB090510 are reported in Abdo et al. (2009), Ghirlanda et al. (2009) and De Pasquale et al. (2009). The high-energy emission above $100 \mathrm{MeV}$ shows a simple power-law decay after the peak, with a decay slope of $\alpha_{\mathrm{LAT}}=-1.38 \pm 0.07$ (De Pasquale et al. 2009) or $-1.46_{-0.03}^{+0.06}$ (Ghirlanda et al. 2009). The XRT and UVOT starts to observe this burst from $100 \mathrm{~s}$ after the burst. The $\mathrm{X}$-ray spectrum is $\beta_{x}=0.57 \pm 0.08$ and the temporal decay index is $\alpha_{x, 1}=-0.74 \pm 0.03$ during the initial $1000 \mathrm{~s}$ and subsequently steepens to $\alpha_{x, 2}=-2.18 \pm 0.10$ with a break at about $t_{b}=1.43 \mathrm{ks}$. The spectrum and temporal decay index of the $\mathrm{X}$-ray emission before $t_{b}$ is well consistent with the forward shock emission with $\nu_{x}<\nu_{c}$ and $p \simeq 2.2$, produced by a spher- 
ical blast wave expanding in a constant density medium. The steeper decay with a slope $\alpha_{x, 2}=2.18 \pm 0.10$ after the break is consistent with a jet break model (Kumar \& Barniol Duran 2009). Such an interpretation predicts a decay slope of $\alpha=\alpha_{x, 1}-\Delta \alpha=-0.99$ at high-energy frequency with $\nu_{*}>\nu_{c}$ in the standard synchrotron scenario, since the different cooling behavior of electrons causes a difference of $\Delta \alpha=0.25$ in the decay slope. This slope is much shallower than the observed slope. The high-energy gamma-ray emission observed from the long burst GRB090902B by Fermi/LAT is reported in Abdo et al. (2009b). LAT detected high-energy gammaray emission above $100 \mathrm{MeV}$ on time scales much longer than the prompt phase. The time-integrated spectrum of the LAT detected emission after the prompt phase is consistent with $\beta_{L A T}=-1.1 \pm 0.1$. Its flux declines as a simple power-law with a decay slope of $\alpha_{L A T}=-1.5 \pm 0.1$ from $t=25 \mathrm{~s}$ to $1 \mathrm{ks}$. Taking $p=-2 \beta_{L A T}=2.2 \pm 0.2$, the standard synchrotron emission predicts a decay slope of $\alpha=-(3 p-2) / 4=-1.15 \pm 0.1$, which is shallower than the observed decay slope, similar to the case in GRB090510. We suggest that one possible origin for the discrepancy in the theoretical and observed decay slopes seen in GRB090510 and GRB090902B is due to the KN effect on high-energy electrons ${ }^{8}$, as discussed in $\S 4$.

The spectrum of the afterglow emission at high-energies can be changed as well when $Y\left(\gamma_{*}\right)$ increases to be large than 1 at later times, since the electron distribution is changed in this case. Therefore, the time-resolved spectrum at highenergies at later times can be harder than $F_{\nu} \sim \nu^{-p / 2}$. However, the time-integrated spectrum of the high-energy emission will be still $F_{\nu} \sim \nu^{-p / 2}$ since the time-integrated fluence is dominated by the contribution at early times (note that the flux decays usually faster than $\left.t^{-1}\right)$, at which times $Y\left(\gamma_{*}\right)<1$ usually. The low-significance data of the time-resolved spectra of GRB090510 and GRB090902B prevent us from identifying the KN effect in the spectrum (De Pasquale et al. 2009; Abdo et al. 2009b) . Obtaining high-significance time-resolved spectra at high-energies from bright GRBs in future would

\footnotetext{
8 A radiative blast wave interpretation for the fast decay has also been proposed recently by Ghisellini et al. (2009). However, a very high $\epsilon_{e}$ (i.e. $\epsilon_{e} \simeq 1$ ) in additional to the fast-cooling spectrum is needed to make the blast
}

be very useful do this.

\section{SUMMARY}

We have studied the KN effect on early high-energy afterglow emission of GRBs. Our findings are summarized as:

i) The IC scatterings between high-energy electrons that produce early-time high-energy ( $~(100 \mathrm{MeV}$ ) afterglow emission and synchrotron peak photons of the afterglow are generally in the deep KN scattering regime. As a result, the IC loss of these electrons is small and the synchrotron luminosity at $h \nu \gtrsim 100 \mathrm{MeV}$ is high, which is favorable for the detection of high-energy gamma-ray emission from the early afterglow by Fermi LAT.

ii)The high-energy gamma-ray emission at the early afterglow phase is dominated by the synchrotron emission. The SSC emission becomes dominated only at energies above the maximum synchrotron photon energy of shock-accelerated electrons, but at such energies the SSC flux is usually too weak to be detectable by Fermi LAT.

iii)The KN suppression effect of high-energy electrons weakens with time, so that the IC loss increases with time. In the parameter space where the Compton parameter is $Y\left(\gamma_{*}\right)>$ 1 , the increasing IC loss leads to a faster temporal decay of the synchrotron afterglow emission at high frequency. The decay slope could be steeper by a factor of $\Delta \alpha=0.5$ at most under favorable conditions. This may explain the rapid decay of the early-time high-energy emission observed in GRB090510 and GRB090902B.

This work is supported by the 973 program under grants 2007CB815404 and 2009CB824800, the NSFC under grants 10973008, 10873009, 10843007, 10503012, 10621303 and 10633040, the Foundation for the Authors of National Excellent Doctoral Dissertations of China, the Qing Lan Project, NCET and the NASA grants (NNX09AT72G and NNX08AL40G).

wave radiative.

\section{REFERENCES}

Abdo, A. et al. 2009a, arXiv:0908.1832

Abdo, A. et al. 2009b, ApJL, in press, arXiv:0909.2470

Abdo, A. et al. 2009c, ApJ, accepted, arXiv:0910.4192

De Pasquale, M., Schady, P., Kuin, N. P. M., 2009, ApJ, arXiv:0910.1629

Dermer, C. D. \& Atoyan, A., 2004, A\&A, 418, L5

Fan, Y. Z. et al., 2008, MNRAS, 384, 1483

Gao, W. H. et al., 2009, ApJL, arXiv:0908.3975

Ghirlanda, G., Ghisellini, G. \& Nava, L., 2009, arXiv:0909.0016

Ghisellini, G., Ghirlanda, G. \& Nava, L., 2009, arXiv:0910.2459

Gou, L. J. \& Mészáros , P., 2007, ApJ, 668, 392

Granot, J. \& Guetta, D., 2003, ApJ, 598, L11

He, H. N., Wang, X. Y., Yu, Y. W. \& Mészáros , P., 2009, ApJ, 706, 1152

Kumar, P. \& Panaitescu, A., 2001, ApJ, 554, 667
Kumar, P. \& Barniol Duran, 2009a, MNRAS, in press, arXiv:0905.2417 Kumar, P. \& Barniol Duran, 2009b, arXiv:0910.5726 Li, Z. \& Waxman, E., 2006, ApJ, 651, 328

Nakar, E., Ando, S. \& Sari, R., 2009, ApJ, 703, 675

Pe'er, A. \& Waxman, E., 2004, ApJ, 603, L1

Sari, R., Piran, T. \& Narayan, R., 1998, ApJ, 497, L17

Sari, R. \& Esin, A. A., 2001, ApJ, 548, 787

Wang, X. Y., Dai, Z. G. \& Lu, T., 2001a, ApJ, 546, L33

Wang, X. Y., Dai, Z. G. \& Lu, T., 2001b, ApJ, 556, 1010

Wang, X. Y., Li, Z., Dai, Z. G., \& Mészáros , P., 2009, ApJ, 698, L98

Wang, X. Y., Cheng, K. S., Dai, Z. G. \& Lu, T., 2005, A\&A, 439, 957

Zhang, B. \& Mészáros , P., 2001, ApJ, 559, 110 